\section{Radiopharmaceuticals in Nuclear Pharmacy and Nuclear Medicine}

\author{
R.J. Kowalsky and K.D. Weatherman
}

Washington, DC: American Pharmacists Association, 2020, 943 pages, $\$ 249.95$

The fourth edition of Radiopharmaceuticals in Nuclear Pharmacy and Nuclear Medicine further builds on the strong foundation of the previous editions. This latest edition reorganizes the 943-page text into 6 sections: introductory materials, physics and radiation safety, radiopharmaceutical chemistry, radiopharmacy practice considerations, regulatory considerations, and clinical applications. Five new chapters have been added that expand on previous-edition subtopics: adverse reactions to radiopharmaceuticals; pregnancy, pediatric, and breastfeeding considerations; mechanisms of localization of radiopharmaceuticals; nonradioactive pharmaceuticals used in nuclear medicine; and PET radiopharmaceutical manufacturing and distribution. The existing chapters also benefit from updates to information as well as additional tables and figures.

The first section consists of 2 introductory chapters. The first is a general overview of the different imaging modalities, nuclear medicine, and nuclear pharmacy. The second is a thorough expansion on the history of nuclear pharmacy from the previous edition. This chapter provides the reader with extensive background information on how the field of nuclear pharmacy was conceived and has evolved over time. Many of the early pioneers of nuclear pharmacy are discussed, along with their contributions to the field.

The second section, on physics and radiation safety, delves into the more familiar material of the previous edition. The chapters in this section cover the principles of radioactivity, radionuclide production, interactions of radiation with matter, radiation detection and instrumentation, radiation safety, and radiation biology and risk. These chapters provide the foundation to understanding the chemical and physical properties of radiopharmaceuticals. The chapter on the principles of radioactivity begins with basic atomic structure and builds through the energy levels and modes of radioactive decay. The next chapter introduces the reader to reactor- and cyclotron-based methods of radionuclide production and how some of that material is incorporated into radionuclide generators. Next is a chapter on interactions of radiation with matter, which sets the stage for subsequent chapters on detection and instrumentation and on radiation safety and biologic risk. The organization of this section is well designed for a logical progression from learning the fundamentals to applying them to more complex concepts.

The third section consists of 4 chapters devoted to radiopharmaceutical chemistry. The first chapter reviews basic chemistry concepts with respect to radiochemistry and radiopharmaceuticals. Important chemistry concepts that are unique to radiopharmaceuticals are discussed, such as radiolabeling and specific activity. The second chapter of this section focuses on the radiopharma-

COPYRIGHT @ 2020 by the Society of Nuclear Medicine and Molecular Imaging. ceutical chemistry of technetium agents. In addition to an extensive discussion on general technetium chemistry, each Food and Drug Administration-approved technetium radiopharmaceutical kit is discussed with regard to normal preparation, radiolabeling mechanisms, and usual dosing. This chapter is one of the most informationally dense and is indispensable to any nuclear medicine professional. The next chapter, entitled, "Radiopharmaceutical Chemistry: Nontechnetium Agents," is a broad-scope chapter that discusses primarily the production, chemistry, and uses of longerlived diagnostic and therapeutic radiopharmaceuticals. Iodine is predominant in this chapter, but the more recently approved lutetium and radium therapeutic radiopharmaceuticals make their debut in this edition. Shorter-lived PET isotopes are covered in the last chapter of this section, entitled, "Radiopharmaceutical Chemistry: PET Agents." This updated chapter takes into consideration the unique nature of PET drugs and PET drug production in describing the terminology, synthesis, and applications of PET radiopharmaceuticals. Similar to the second chapter in this section, this chapter thoroughly discusses individual PET radiopharmaceuticals. The information on quality control and quality assurance for PET radiopharmaceuticals has been expanded to give the reader a greater depth of knowledge about the production of these drugs.

Section 4, on radiopharmacy practice considerations, describes the day-to-day operation of the nuclear pharmacy, whether it be a centralized commercial pharmacy or a pharmacy housed within a large institution. The first chapter provides a global overview of the facilities, equipment, and personnel needed to operate a commercial nuclear pharmacy. The second chapter discusses the vital function of quality control of radiopharmaceuticals as part of the overall quality assurance of the finished product. Methods of analysis for chemical, pharmaceutical, and biologic considerations are discussed at length, with figures that help the reader understand the separation processes. The next chapter, on radiopharmaceutical preparation problems, is an expansion of a subtopic in the previous edition. This chapter discusses several chemical reaction issues that can occur in the preparation of radiopharmaceuticals. The information supplements the previous chemistry chapters and functions as a troubleshooter to failures in radiopharmaceutical kit preparation. The fourth chapter in this section, on radiolabeled blood products, is a reorganization and consolidation of information from the previous edition. After presenting a brief history of blood product radiolabeling, this chapter discusses the isolation, radiolabeling, and quality control testing of leukocytes. Labeling of other blood products, such as red blood cells, is discussed in a different chapter because such labeling is generally performed at the imaging facility rather than at the centralized nuclear pharmacy. The final chapter in this section is on PET radiopharmaceutical manufacturing and distribution. This chapter builds on what the reader learned about the radiopharmaceutical chemistry of PET agents in a previous chapter and how these radiopharmaceuticals have their own unique production and distribution characteristics. This chapter also includes detailed background on Food and Drug Administration and PET regulatory issues and provides a seamless transition into the next section, on regulatory control.

As cumbersome as regulations can be for radioactive materials, they are necessary for the health and safety of those handling the 
materials, the general public, and the patients. Regulatory considerations are the topic of the fifth section of the book, containing 3 chapters. The first, entitled, "Licensing and Regulatory Control: Radioactivity and Radioactive Materials," provides up-to-date information on Nuclear Regulatory Commission licensing and regulatory control of radioactive materials pertaining to diagnostic and therapeutic radiopharmaceuticals. The next chapter, entitled, "Licensing and Regulatory Control: Radiopharmaceutical Oversight," discusses the regulatory evolution of radiopharmaceuticals, with a primary focus on Food and Drug Administration oversight at the federal level. These 2 chapters help to define the oftenconfusing but important regulatory differences in the drug and radioactive components of radiopharmaceuticals. The final chapter of this section discusses the mechanisms of radiopharmaceutical localization. Although the material is not regulation-based, this chapter provides a transition to the clinical section by discussing how various radiopharmaceuticals localize to their intended targets. Several figures in this chapter illustrate biodistribution via these processes.

Clinical applications are the topic of the sixth and final section of the book, containing 13 chapters. The restructuring of the new edition includes 2 new chapters in this section, the first of which is on considerations relating to pregnancy, pediatrics, and breastfeeding - a welcome upgrade from a subtopic in the previous edition. The second new chapter is another subtopic breakout, on adverse reactions to radiopharmaceuticals. The next chapter, on nonradioactive pharmaceuticals used in nuclear medicine, discusses adjunct pharmaceuticals used as part of a nuclear medicine study. These pharmaceuticals are also discussed in their respective chapters of use, but this chapter provides a nice consolidation and somewhat more detail on mechanism, dosage, and patient management concerns.

The next 7 chapters of this section describe the clinical uses of radiopharmaceuticals in specific organs or organ systems. All chapters are refreshed with updated material, figures, and tables. There are many more images and colored overlays in this edition that help the reader visualize how the data are obtained and analyzed. Following the specific organ system chapters is a chapter on total-body SPECT and related procedures, which focuses on whole-body, soft-tissue studies for infection and tumor imaging. Updated information in this chapter includes the recently approved ${ }^{68} \mathrm{Ga}$-DOTATATE for neuroendocrine tumors and the highly anticipated prostate-specific membrane antigen-targeting ligands for prostate cancer. Next is a chapter on PET whole-body imaging procedures. This chapter includes an extensive discussion of the biodistribution of ${ }^{18} \mathrm{~F}-\mathrm{FDG}$ in normal tissues and on its clinical uses in a variety of cancers. The remainder of the chapter is dedicated to nononcologic imaging procedures such as for the heart, epilepsy, dementia, $\beta$-amyloid, and prostate cancer with ${ }^{18} \mathrm{~F}$-FDG or alternative PET radiopharmaceuticals. The final chapter of this section is on therapeutic applications of radioactive agents. This chapter starts with the older therapeutic radiopharmaceuticals such as strontium and samarium and transitions to the newer generation of therapeutic radiopharmaceuticals such as yttrium, radium, and lutetium, with discussion of the approach to using small molecules to bind to specific receptors for delivery of targeted radiotherapy.

Radiopharmaceuticals in Nuclear Pharmacy and Nuclear Medicine is a well-written and well-organized textbook that builds on the success of the previous editions. The text is versatile enough to be understood by students and effective enough to serve as an essential reference for the professional practitioner or to help those preparing for board certification testing. This textbook continues to justify its position as one of the best references in the field and should be included in the library of any nuclear medicine or radiopharmacy practice.

\author{
Nic Mastascusa, PharmD, RPh, BCNP \\ University of Iowa Hospitals and Clinics \\ 3974 JPP 200, Hawkins Dr. \\ Iowa City, IA 52242 \\ E-mail: nmastasc@healthcare.uiowa.edu
}

Published online Jul. 24, 2020.

DOI: $10.2967 /$ jnmt.120.253674 